\title{
HARDY TYPE INEQUALITIES AND COMPACTNESS OF A CLASS OF INTEGRAL OPERATORS WITH LOGARITHMIC SINGULARITIES
}

\author{
Akbota M. Abylayeva And LARS-ERIK PERSSON
}

Abstract. We establish criteria for both boundedness and compactness for some classes of integral operators with logarithmic singularities in weighted Lebesgue spaces for cases $1<p \leqslant q<$ $\infty$ and $1<q<p<\infty$. As corollaries some corresponding new Hardy inequalities are pointed out.

Mathematics subject classification (2010): 47G10, 47B38, 45P05.

Keywords and phrases: Inequalities, Hardy type inequalities, singular integral operator, logarithmic singularity, fractional integration operator, boundedness, compactness.

\section{REFERENCES}

[1] A. M. Abylayeva And M. Zh. OMirbeK, A weighted estimate for integral operator with a logarithmic singularity, Izv. Nats. Akad. Nauk. Resp. Kaz. Ser. Fiz. Mat. 1, (2005), 38-47 (in Russian).

[2] A. M. Abylayeva And A. O. Baiarystanov, Compactness criterion for fractional integration operator of infinitesimal order, Ufa Math. J., 5, 1 (2013), 3-10.

[3] K. F. ANDERSEN AND E. T. SAWYER, Weighted norm inequalities for the Riemann-Liouville and Weyl fractional integral operators, Trans. Amer. Math. Soc., 308, 2 (1988), 547-558.

[4] V. I. BURENKOV AND R. OINAROV, Necessary and sufficient condition for boundedness of the Hardytype operator from a weighted Lebesgue space to a Morrey-type space, Math. Inequal. Appl., 16, 1 (2013), 1-19.

[5] E. M. DYNKIN AND B. P. OsILENKER, Weighted estimates for singular integrals and their applications, Mathematical analysis. Itog: Nauki i Tekhniki, Acad. Nauk. SSSR, Vsesoyuz. Inst. Nouchn. Inform., Moscow, 21, (1982), 42-129.

[6] D. E. Edmunds, V. Kokilashvili And A. Meskhi, Bounded and compact integral operators, Kiluwer Academic Published., XVI, Boston/Dordrecht/London, 2002.

[7] S. M. FARS ANI, On the boundedness and compactness of the fractional Riemann-Liouville operators, Sibirsk. Mat. Zh., 54, 2 (2013), 468-479 (in Russian); translation in Sib. Math. J. 54 (2013), no. 2, 368-378.

[8] A. Kalybay, On boundedness of the conjugate multidimensional Hardy operator from a Lebesgue space to a local Morrey-type space, Int. J. Math. Anal., 8, 9-12 (2014), 539-553.

[9] L. V. Kantorovich And G. R. AkIlov, Functional analysis, Oxford etc.: Pergamon Press XIV, 1982.

[10] M. A. Krasnosel'skit, P. P. Zabreiko, E. N. Pustilnik and P. E. Sobolewski, Integral operators in spaces of summable functions, Moscow: Nauka, 1966 (in Russian).

[11] A. Kufner, L. Maligranda And L. E. Persson, The Hardy Inequality. About its History and Some Related Results, Vydavatelsky Servis Publishing House, Pilsen, 2007.

[12] A. Kufner And L. E. Persson, Weighted Inequalities of Hardy Type, World Scientific, New Jersey/London/Singapore/Hongkong, 2003.

[13] A. Kufner, L. E. Persson And N. Samko, Hardy type inequalities with kernels: the current status and some new results, Math. Nachr. (to appear).

[14] V. KokilashVili, A. Meskhi And L. E. Persson, Weighted Norm Inequalities for Integral Transforms with Product Kernels, Nova Scientific Publisher, INC., New York, 2009. 
[15] A.MeSKHI, Solution of some weight problems for the Riemann-Liouville and Weil operators, Georgian Math. J. 5 (1998), no. 6, 565-574.

[16] A. M. NAKhusheV, Equations of mathematical biology, M. Vysshaya Shkola, 1995 (in Russian).

[17] R. OINAROv, Boundedness of integral operators from weighted Sobolev space to weighted Lebesgue space, Complex Var. and Elliptic Equ. 56 (2011), no. 10-11, 1021-1038.

[18] R. OINAROV, Boundedness and compactness of Volterra type integral operators, Sibirsk Math. Zh. 48 (2007), no. 5, 1100-1115 (in Russian); translation in Siberian Math. J. 48 (2007), no. 5, 884-896.

[19] B. OpIC And A. Kufner, Hardy type inequalities, Pitman Research Notes in Mathematics, John Wiley, New York, (1990).

[20] D. V. PROKHOROV, On the boundedness and compactness of a class of integral operators, J. London Math. Soc. 61 (2000), no. 2, 617-628.

[21] D. V. Prokhorov And V. D. Stepanov, Weighted estimates for the Riemann-Liouville Operators and Applications, Tr. Mat. Inst. Steklova 243 (2003), 289-312 (in Russian). 